\title{
Identification and taxonomy of some entomopathogenic Paecilomyces spp. (Ascomycota) isolates using rDNA-ITS Sequences
}

\author{
Peter W. Inglis and Myrian S. Tigano \\ Embrapa Recursos Genéticos e Biotecnologia, Parque Estação Biológica, Brasília, DF, Brazil.
}

\begin{abstract}
A phylogenetic analysis of the 5.8S rDNA and internal transcribed spacer (ITS1 and ITS2) sequences from some entomogenous Paecilomyces species supports the polyphyly of the genus and showed the existence of cryptic species. In the Eurotiales, anamorphs Paecilomyces variotii and Paecilomyces leycettanus were related to the teleomorphs Talaromyces and Thermoascus. In the Hypocreales, three major ITS subgroups were found, one of which included Paecilomyces viridis, Paecilomyces penicillatus, Paecilomyces carneus and isolates identified as Paecilomyces lilacinus and Paecilomyces marquandii. However, the majority of the $P$. lilacinus and $P$. marquandii isolates formed a distinct and distantly related subgroup, while the other major subgroup contained Paecilomyces farinosus, Paecilomyces amoeneroseus, Paecilomyces fumosoroseus and Paecilomyces tenuipes.
\end{abstract}

Key words: Paecilomyces spp., phylogenetic analysis, rDNA; molecular taxonomy.

Received: January 25, 2005; Accepted: May 31, 2005.

The hyphomycete genus Paecilomyces was revised by Samson (1974), who recognized and defined 31 species and divided the genus into two sections. Section Paecilomyces contains members which are often thermophilic, the perfect states being placed in the ascomycetous genera Talaromyces and Thermoascus. Section Isarioidea contains mesophiles, including several well-known entomopathogenic or nematophagous species such as Paecilomyces farinosus, Paecilomyces fumosoroseus, Paecilomyces amoeneroseus, Paecilomyces lilacinus, Paecilomyces javanicus and Paecilomyces tenuipes. Classification of these fungi is based on morphological characteristics, which are often highly subjective, with unambiguous identification to the species level often being extremely difficult. Additionally, the morphological differences observed may be the product of simple mutations or media/cultivation effects and thus are not always reliable characters. Definitions of some entomopathogenic or nematophagous species in the genus Paecilomyces are subject to doubt when classical identification methods are compared to analysis of genetic markers (Tigano-Milani et al., 1995a; Tigano-Milani et al., 1995b; Cantone and Vandenberg, 1998). The limitations of traditional identification techniques indicate that alternative methods need to be developed for the identification of these fungi.

Send correspondence to P.W. Inglis. Embrapa Recursos Genéticos e Biotecnologia, Parque Estação Biológica, Av. W/5 Norte Brasília, 70770900 Brasília, DF, Brazil. E-mail: peteringlis@onetel. com.
Comparative analysis of ribosomal RNA (rDNA) gene sequence information can be used to clarify natural evolutionary relationships over a wide taxonomic range (Pace et al., 1986). Ribosomal RNA genes (rDNA) typically exist as a tandem repeat that includes coding regions, which are conserved to varying degrees, as well as highly divergent spacer regions. These spacer regions, or internal transcribed spacer sequences (ITS), have been widely used in fungal systematics (Bowman et al., 1992; Hibbett, 1992; Driver et al., 2000). In the case of the genus Paecilomyces, the analysis of sequences of the large and the small subunit rRNA gene has already indicated the polyphyly of the genus (Obornik et al., 2001). We therefore decided to use rDNA-ITS sequencing to clarify the taxonomic relationships and identities of some entomopathogenic and nematophagous Paecilomyces isolates held in our culture collection.

For our analysis we selected a large number of entomopathogenic Paecilomyces isolates, including several that could not be unambiguously assigned to a known species using morphological characters (Table 1). Monosporic fungal cultures were prepared from liquid nitrogen stored or lyophilized stocks from the Collection of Entomopathogenic Fungi held at Embrapa - Recursos Genéticos e Biotecnologia, Brasília-DF, Brazil or the Insect Pathogen Culture Collection of the Commonwealth Scientific and Industrial Research Organization (CSIRO) Division of Entomology (DE-CSIRO), Canberra Australia). 
Table 1 - Origin and conventional classification of isolates, whose ITS1-5.8S-ITS2 DNA was sequenced in this study.

\begin{tabular}{|c|c|c|c|c|c|}
\hline $\begin{array}{l}\text { Species designation } \\
\text { (classical or as received) }\end{array}$ & Isolate $^{a}$ & Host or substrate & Country & Year & Isogenic group \\
\hline Nomuraea anemenoides & IMI214110 & Soil & Australia & 1974 & \\
\hline Nomuraea rileyi & CG129 & Anticarsia gemmatalis & Brazil (DF) & 1988 & \\
\hline P. farinosus & CBS541.81 & Spider & Ecuador & 1981 & \\
\hline P. farinosus & CG199 & Lymantria dispar & USA & 1986 & \\
\hline P. fumosoroseus & ARSEF1576 & Monophadnus sp. & Italy & 1984 & \\
\hline P. fumosoroseus & CG170 & Pseudococcus sp. & USA (Florida) & 1990 & \\
\hline P. fumosoroseus & CG196 & Diptera, Tachnindae & Poland & 1983 & \\
\hline P. fumosoroseus & CG204 & Bemisia $\mathrm{sp}$ & Mexico & 1990 & \\
\hline P. fumosoroseus & CG325 & Nilaparvata lugens & Philippines & 1985 & \\
\hline P. fumosoroseus & CG335 & Soil & Brazil (MT) & 1990 & \\
\hline P. fumosoroseus & CG741 & Lagria villosa & Brazil (GO) & 1982 & \\
\hline P. javanicus & CBS134.22 & & Ecuador & 1922 & \\
\hline P. marquandii & ARSEF3047 & Soil & Mali & & \\
\hline P. marquandii & IMI2233 & Meloidogyne sp. & India & & \\
\hline Paecilomyces carneus & CG525 & Soil & Brazil (RS) & 1995 & \\
\hline Paecilomyces carneus & IMI058418 & Soil & UK & & \\
\hline Paecilomyces leycettanus & IMI178525 & & & & \\
\hline Paecilomyces penicillatus & IMI186962 & Mushroom & Belgium & & \\
\hline Paecilomyces sp. & CG177 & Meloidogyne sp. & Brazil (PA) & 1988 & \\
\hline Paecilomyces variotti & CG503 & Air & Brazil (SP) & 1995 & \\
\hline Paecilomyces variotti & FRR3797 & Soil & Brazil & & \\
\hline Paecilomyces viridis & ARSEF2456 & & France & & \\
\hline Talaromyces leycettanus & FRR3525 & & & & \\
\hline Trichoderma viridis & ARSEF807 & & & & \\
\hline P. lilacinus & CG265 & Soil & Brazil (TO) & 1991 & A \\
\hline P. lilacinus & CG301 & Coleoptera & Brazil (RJ) & 1985 & A \\
\hline P. lilacinus & CG348 & Soil & Brazil (GO) & 1991 & A \\
\hline P. fumosoroseus & CG123 & Spaethiella sp. & Brazil (AM) & 1987 & $\mathrm{~B}$ \\
\hline P. fumosoroseus & CG197 & Pyrrhalta luteola & France & 1983 & $\mathrm{~B}$ \\
\hline P. fumosoroseus & CG397 & Bemisia tabaci & USA (Florida) & 1992 & $\mathrm{~B}$ \\
\hline P. fumosoroseus & CG404 & Bemisia tabaci & Nepal & 1992 & $\mathrm{~B}$ \\
\hline Paecilomyces sp. & CG499 & Coleoptera & Brazil (DF) & 1995 & B \\
\hline P. fumosoroseus & CG684 & Bombyx mori & China & 1981 & B \\
\hline P. fumosoroseus & FI1217 & & & & $\mathrm{B}$ \\
\hline P. amoeneroseus & CBS738.73 & Coleoptera & Ghana & 1973 & $\mathrm{C}$ \\
\hline P. amoeneroseus & CG162 & Lagria villosa & Brazil (GO) & 1989 & $\mathrm{C}$ \\
\hline P. amoeneroseus & CG163 & Lagria villosa & Brazil (GO) & 1989 & $\mathrm{C}$ \\
\hline$P$. fumosoroseus & CG302 & Lagria villosa & Brazil (GO) & 1982 & $\mathrm{C}$ \\
\hline P. fumosoroseus & ARSEF1005 & Bombyx mori & Japan & 1998 & $\mathrm{D}$ \\
\hline P. fumosoroseus & ARSEF1506 & Pyrrhalta luteola & France & 1983 & $\mathrm{D}$ \\
\hline P. fumosoroseus & ARSEF1644 & Musca domestica & France & 1984 & $\mathrm{D}$ \\
\hline P. fumosoroseus & ARSEF1645 & Musca domestica & France & & $\mathrm{D}$ \\
\hline P. fumosoroseus & ARSEF2679 & Popillia japonica & Portugal & & $\mathrm{D}$ \\
\hline P. fumosoroseus & ARSEF2745 & Soil & Philippines & & $\mathrm{D}$ \\
\hline P. fumosoroseus & ARSEF2749 & Plutella xylostella & Philippines & & $\mathrm{D}$ \\
\hline P. fumosoroseus & ARSEF887 & Diptera & France & & $\mathrm{D}$ \\
\hline P. tenuipes & ARSEF2489 & Spodoptera frugiperda & Mexico & & $\mathrm{E}$ \\
\hline P. tenuipes & ARSEF2490 & Spodoptera frugiperda & Mexico & & $\mathrm{E}$ \\
\hline P. tenuipes & ARSEF2491 & Spodoptera frugiperda & Mexico & & $\mathrm{E}$ \\
\hline P. tenuipes & IMI180610 & Lepidoptera & Netherlands & & $\mathrm{E}$ \\
\hline P. lilacinus & CBS284.36 & Soil & USA & 1936 & $\mathrm{~F}$ \\
\hline P. lilacinus & CG36 & Deois flavopicta & Brazil (DF) & 1992 & $\mathrm{~F}$ \\
\hline P. lilacinus & CG189 & Soil & Brazil (GO) & 1991 & $\mathrm{~F}$ \\
\hline P. lilacinus & CG267 & Soil & Brazil (GO) & 1991 & $\mathrm{~F}$ \\
\hline P. lilacinus & CG275 & Soil & Brazil (MA) & 1991 & $\mathrm{~F}$ \\
\hline P. lilacinus & CG299 & Soil & USA (Florida) & 1990 & $\mathrm{~F}$ \\
\hline P. lilacinus & CG190 & Soil & Brazil (RS) & 1990 & G \\
\hline P. lilacinus & CG271 & Soil & Brazil (GO) & 1991 & G \\
\hline
\end{tabular}

${ }^{a}$ Isolate accession numbers from their appropriate culture collection are denoted by the following prefixes: ARSEF, Agricultural Research Service Entomopathogenic Fungus Collection, USDA, Ithaca, NY, USA; CBS, Centraalbureau voor Schimmelcultures, Utrecht, The Netherlands; CG, Embrapa Collection, Brasília, Brazil; FI, CSIRO Collection, Canberra, Australia; IMI, International Mycological Institute, Surrey, UK. Isolates with identical ITS1-5.8S-ITS2 gene sequences were assigned to an appropriate isogenic group and are represented in Figure 1 by this group designation. Key to Brazilian states: $\mathrm{AM}=$ Amazonas, $\mathrm{DF}=$ Distrito Federal, $\mathrm{GO}=$ Goiás, MA = Maranhão, MT = Mato Grosso, PA = Pará, RJ = Rio de Janeiro, RS = Rio Grande do Sul, TO = Tocantins. 
Genomic DNA was purified from mycelium using the cetyltrimethylammonium bromide (CTAB) extraction method of Rogers and Bendich (1988). A single pair of primers was used to PCR amplify the internal transcribed spacer (ITS) regions ITS1 and ITS2, along with the central 5.8S rDNA, essentially as described by Driver et al. (2000). The primers used were the universal eukaryotic ITS primers, which annealed to the 3 ' end of the 16/18S rDNA (TW81; 5'GTTCCGTAGGTGAACCTGC) and to the 5' end of the 28S rDNA (AB28; 5'ATATGCTTAAGTTCA GCGGGT) respectively (White et al., 1990). The PCR products were checked using agarose gel electrophoresis and, as expected, produced a product of about $500 \mathrm{bp}$. Bands were excised and then purified using the Geneclean II kit (Bio 101) and were either cloned using the PCRscript-Amp plasmid vector/cloning kit (Stratagene) prior to sequencing or were sequenced directly using consensus primers. Sequencing of both strands of the PCR products/clones was as described previously (Driver et al., 2000).

The ITS1-5.8S-ITS2 gene sequences were aligned with ClustalX v. 1.83.1 (Thompson et al. 1997). Selected sequences available from GenBank were also included in the analysis (Table 1). A Maximum Likelihood with molecular clock tree of divergence data from the ITS alignments was calculated using the DnaMLK program from PHYLIP v. 3.6 (Felsenstein and Churchill, 1996).

The phylogenetic tree, summarizing the relationships between the ITS sequences obtained or used in this study (Figure 1), possesses a major division corresponding to the Ascomycete orders Eurotiales and Hypocreales. These orders respectively, contain members of the two sections of Paecilomyces proposed by Samson (1974), where section Paecilomyces is found in the Eurotiales (Trichocomaceae) and section Isarioidea in the Hypocreales (Clavicipitaceae and Hypocreaceae) (Figure 1). Our ITS analysis data supports the hypothesis that Paecilomyces is polyphyletic, which has been previously suggested using rDNA data (Obornik et al., 2001) and supports the morphological division of the genus into these two sections. Section Paecilomyces, with the type species Paecilomyces variotii, contains thermophiles with affinities to Talaromyces and Byssochlamys, which are related to the class Plectomycetes in the Ascomycota. Section Isarioidea, with the type species $P$. farinosus, are mesophiles, mostly entomogenous and with affinities to the order Clavicipitales in the class Pyrenomycetes within the Ascomycota (Mugnier, 1998; Samson, 1974).

The Eurotiales group included a clade containing all Paecilomyces variotii isolates. These strains then join a group comprising Byssochlamys nivea, Thermoascus crustaceus, Paecilomyces leycettanus and Talaromyces leycettanus, which then joins a separate group comprising Talaromyces purpureus, Nomuraea anemenoides and Penicillium minioluteum. The presence of Talaromyces



Figure 1 - Phylogenetic tree for Paecilomyces species based on internal transcribed spacer (ITS) and 5.8S rDNA sequences using the Maximum Likelihood with molecular clock method. The ascomycetous classes are indicated on the major branches. Sequence divergence is indicated by the scale bar. Samples giving identical ITS1-5.8S-ITS2 gene sequences were assigned to an arbitrary group and are represented in the tree by their group/species designation. Strains marked with an asterisk* are GenBank accessions not sequenced in the study. The tree and its data matrix have been submitted to TREEBASE.

and Thermoascus in the same clade as Paecilomyces leycettanus and $P$. variotii supports the relationship of these anamorph species from section Paecilomyces to these teleomorph genera. Species of Talaromyces were present in both subgroups of the Eurotiales, where some Talaromyces species are more closely related to Byssochlamys than others. In fact, these data agree with other studies showing that Penicillium and Talaromyces are not monophyletic genera (Berbee et al., 1995; LoBuglio et al., 1993). The Talaromyces leycettanus ITS sequence was a perfect match with $P$. leycettanus, which can therefore be considered the teleomorph for this Paecilomyces species. The Hypocreales group possesses a topology that suggests that this group is also not monophyletic. Molecular phylogenetic analyses based on $18 \mathrm{~S}$ rDNA sequences demonstrated that $P$. tenuipes belongs to the Clavicipitales in the class Pyrenomycetes (Fukatsu et al., 1997). Entomogenous species of Paecilomyces are also known to be closely related to 
other insect-pathogenic fungi, Beauveria bassiana and some Verticillium species (Oborník et al., 2001).

The ITS region was found to be useful in resolving some difficulties within classical Paecilomyces taxonomy and redefined the classification of some of our isolates, where a high level of variability among isolates was previously seen based on analysis of other DNA markers (Tigano-Milani et al., 1995a; Tigano-Milani et al., 1995b). Isolates identified morphologically as $P$. lilacinus and $P$. marquandii were located in two, quite distant subgroups. One clade included P. marquandii isolate ARSEF3047, which was very close to unidentified isolate CG177, and an isogenic group of three $P$. lilacinus isolates (group A) which may represent a cryptic species closely related to $P$. carneus. This cluster then joined Paecilomyces viridis ARSEF2456, which appears to be related to Nomuraea rileyi and, to a lesser extent, Metarhizium anisopliae. Other isolates originally identified as $P$. lilacinus, including isogenic isolates of Group F (which includes the type strain CBS284.36) and Group G, formed a distinct clade along with Paecilomyces nostocoides and Cordyceps sinensis. An isolate designated as a 'true' $P$. marquandii strain (IMI2233) was in this group and produced colonies with yellow undersides, a diagnostic trait consistent with the original description of this species (Samson, 1974). This second ITS clade of $P$. lilacinus and $P$. marquandii isolates was in fact more closely related to Trichoderma, Hypocrea and Gliocladium than to the other entomopathogenic Paecilomyces species in the Hypocreales.

A large group, containing $P$. farinosus (the type species for the Isarioidea section), also contained the other well-known entomogenous species $P$. amoeneroseus, $P$. fumosoroseus and $P$. tenuipes. A mycoinsecticide of significant commercial interest for the control of whiteflies is produced using $P$. fumosoroseus (Lacey et al., 2001) but to exploit the considerable potential of this fungus a reliable and standardized approach to its identification is needed. Our study confirmed that $P$. fumosoroseus lacks a discrete species concept, as has already been suggested based on arbitrarily primed PCR and tRNA fingerprinting (TiganoMilani et al., 1995a) and vegetative compatibility (Cantone and Vandenberg, 1998). A previous phylogenetic analysis of $P$. fumosoroseus using rDNA-ITS sequences showed that there are at least three monophyletic groups within the $P$. fumosoroseus complex and that host selective pressure may be significant in the selection of genotypes (Fargues et al., 2002). We found that eight isolates originally classified as $P$. fumosoroseus (represented on the tree by Group D $P$. fumosoroseus) have a similar but distinct ITS sequence to that of the $P$. tenuipes isolates (Group E). These small but consistent differences justify the consideration of these isolates as a different and unknown species, especially considering that the degree of divergence between these isolates and $P$. tenuipes was similar to that obtained between the other $P$. fumosoroseus and $P$. amoeneroseus or $P$. farinosus. In order to obtain more information about their identification these unidentified isolates, which are confused with $P$. fumosoroseus, should be compared to other described Paecilomyces species not analyzed in our study. We found six different ITS sequences among the twelve $P$. fumosoroseus isolates closely related to $P$. amoeneroseus (Group C) and an unidentified isolate (CG499) within this cluster had an ITS sequence identical to that of five $P$. fumosoroseus isolates (Group B). Moreover, a misidentified $P$. fumosoroseus isolate (CG302) possessed an identical sequence to the three $P$. amoeneroseus analyzed (Table 1). This group was then joined by $P$. javanicus and Paecilomyces penicillatus. These results emphasize the difficulties in using classical morphological traits for taxonomy and the unambiguous identification of these closely related species.

Our results confirm the polyphyly of the genus Paecilomyces, already observed by analysis of the large and small-subunit rRNA gene sequences (Obornik et al., 2001; Luangsa-ard et al., 2004), where Paecilomyces probably represents a form genus only. A major review of the genus Paecilomyces is required, including the analysis of a more conserved gene region to clarify the phylogenetic relationships.

\section{Acknowledgments}

This work was supported by a grant to MST from the International Centre for Genetic Engineering and Biotechnology (ICGEB). Additional funding was provided by Embrapa and CNPq. We are indebted to Dr Felice Driver for invaluable practical assistance and insightful advice in this project and also wish to thank Richard Milner and John Truman for the provision of strains and for helpful discussions during the preparation of this manuscript.

\section{References}

Berbee ML, Yoshimura A, Sugiyama J and Taylor JW (1995) Is Penicillium monophyletic? An evolution of the phylogeny in the family Trichocomaceae from $18 \mathrm{~S}, 5.8 \mathrm{~S}$ and ITS ribosomal DNA sequence data. Mycologia 87:210-222.

Bowman BH, Taylor JW, Brownlee AG, Lee J, Lu SD, and White TJ (1992) Molecular evolution of the fungi: relationships of the Basidiomycetes, Ascomycetes and Chytridiomycetes. Mol Biol Evol 9:285-296.

Cantone FA and Vandenberg D (1998) Intraspecific diversity in Paecilomyces fumosoroseus. Mycol Res 102:209-215.

Driver F, Milner RJ and Trueman JWH (2000) A taxonomic revision of Metarhizium based on a phylogenetic analysis of rDNA sequence data. Mycol Res 104:143-150.

Fargues J, Bon MC, Manguin S and Couteaudier Y (2002) Genetic variability among Paecilomyces fumosoroseus isolates from various geographical and host insect origins based on the rDNA-ITS regions. Mycol Res 106:1066-1074.

Felsenstein J and Churchill GA (1996) A hidden Markov model approach to variation among sites in rate of evolution. Mol Biol Evol 13:93-104. 
Fukatsu T, Sato H and Kuriyama H (1997) Isolation, inoculation to insect host, and molecular phylogeny of an entomogenous fungus Paecilomyces tenuipes. J Invertebr Pathol 70:203208.

Hibbett DS (1992) Ribosomal RNA and fungal systematics. Trans Mycol Soc Jap 33:533-556.

Lacey LA, Frutos R, Kaya HK and Vail P (2001) Insect pathogens as biological control agents: Do they have a future? Biol Control 21:230-248.

Lo Buglio KF, Pitt JI and Taylor JW (1993) Phylogenetic analysis of two ribosomal DNA regions indicates multiple independent losses of a sexual Talaromyces state among asexual Penicillium species in subgenus Biverticillium. Mycologia 85:592-604.

Luangsa-ard JJ, Hywel-Jones NL and Samson RA (2004) The polyphyletic nature of Paecilomyces sensu lato based on 18S-generated rDNA phylogeny. Mycologia 96:773-780.

Mugnier J (1998) Molecular evolution and phylogenetic implications of ITS sequences in plants and in fungi. In: Bridge P, Couteaudier Y and Clarkson J (eds) Molecular Variability of Fungal Pathogens. CAB International, Wallingford, pp 253-270.

Obornik M, Jirku M and Dolezel D (2001) Phylogeny of mitosporic entomopathogenic fungi: Is the genus Paecilomyces polyphyletic? Can J Microbiol 47:813-819.
Pace NI, Olsen GI and Woese CR (1986) Ribosomal RNA phylogeny and the primary lines of evolutionary descent. Cell 45:325-326.

Rogers SO and Bendich AJ (1988) Extraction of DNA from plant tissues. In: Gelvin SB, Schilperpoort RA and Verma DPS (eds) Plant Molecular Biology Manual. v. A6, Kluwer Academic Publishers, Dordrecht, pp 1-10.

Samson RA (1974) Paecilomyces and some allied hyphomycetes. Studies in Mycology 6. Centraalbureau voor Schimmelcultures, Baarn, 116 pp.

Thompson JD, Gibson TJ, Plewniak F, Jeanmougin F and Higgins DG (1997) The ClustalX windows interface: Flexible strategies for multiple sequence alignment aided by quality analysis tools. Nucleic Acids Res 24:4876-4882.

Tigano-Milani MS, Honeycutt RJ, Lacey L, Assis R, McClelland $M$ and Sobral BWS (1995a) Genetic variability of Paecilomyces fumosoroseus isolates revealed by molecular markers. J Invertebr Pathol 65:274-282.

Tigano-Milani MS, Samson RA, Martins I and Sobral BWS (1995b) DNA markers for differentiating isolates of Paecilomyces lilacinus. Microbiology 141:239-245.

White TJ, Bruns TD, Lee SB and Taylor JW (1990) Analysis of phylogenetic relationships by amplification and direct sequencing of ribosomal genes. In: Innis MA, Gelfand DH, Sninsky JJ and White TJ (eds) PCR Protocols. Academic Press, New York, pp 315-22.

Associate Editor: Darcy Fontoura de Almeida 